\title{
X-RAY ANALYSIS GROUP OF THE INSTITUTE OF PHYSICS AUTUMN CONFERENCE
}

\begin{abstract}
A CONFERENCE and exhibition organized by the X-ray Analysis Group of the Institute of Physics were held in London at the Institution of Electrical Engineers during November 20-21. The conference was concerned with two principal topics: a critical examination of photographic and counter spectrometer methods together with a comparison of these two experimental approaches, and a discussion of X-ray methods of identification.
\end{abstract}

Dr. R. H. Herz, who considered the fundamentals of the behaviour of photographic emulsions, outlined the Gurney-Mott concept of latent image formation : the fact that at least one grain of silver bromide is rendered developable per X-ray quantum absorbed is responsible for the linearity of the initial part of the density-exposure curve. The contrast of all emulsions, whatever their speed, is the same over this linear portion. The speed of the film increases at higher densities, and it also increases with $\mathrm{X}$-ray wave-length. Both contrast and speed can be increased at the expense of heavier fogging by lengthening the time of development.

The requirements of $\mathrm{X}$-ray films for various types of diffraction work were discussed by Dr. P. B. Hirsch, who said that the accuracy of the measurement of the position of diffraction lines is affected by non-uniform shrinkage; spacings cannot generally be determined to better than 2 parts in $10^{5}$ unless a number of calibration marks are made on the camera. For the measurement of weak lines, speed and contrast are of importance. While film speeds are adequate for softer radiation, intensifying sereens give a very desirable increase for molybdenum $K \alpha$ radiation. The visibility of the lines against the background is determined by film contrast, and it is thus improved at higher densities. Under favourable conditions, lines 1 per cent above background are detectable. For the accurate measurement of intensity, fluctuations of the background must be kept to a minimum, and these are more often due to stains originating during development and to impurities in the emulsion than to film graininess. A further cause was shown in the subsequent discussion to be the non-uniformity of the black paper often used to cover the film. The greatest bar to accuracy in intensity measurements is the variation from point to point of the film speed, and differences as great as 5 per cent can occur. Dr. Hirsch emphasized the need for a single-coated X-ray film, not at present available in Britain, a point on which he was strongly supported in the subsequent discussion.

Dr. C. F. Brown next surveyed some of the many types of microdensitometers. For powder work the best available design is probably based on Dobson's split-beam principle, of which a modern version is due to A. Taylor. Among microdensitometers designed for single crystal work, Dr. Brown described in detail a scanning instrument, on show in the exhibition, which is a development of a design by J. M. Robertson and R. H. V. M. Dawton. Incorporating an 'anti-log' circuit, the instrument gives a deflexion on a meter which is directly proportional to the intensity of the spot on the film. Sir Lawrence Bragg suggested that, instead of determining the light transmitted by a film, one could measure the light scattered by the developed silver grains, as had been proposed by J. C. M. Brentano. Dr. R. L. Gordon then compared the relative merits of film and counter spectrometer methods. The expense and complexity of the latter method can, in general, be justified only when a considerable volume of work is contemplated, and Dr. Gordon described his own installation, which makes use of a monitoring counter and can be used with either scalers or counting-rate meters and chart recorders. For qualitative analysis, the positions of lines can be determined very rapidly with an accuracy sufficient for the use of the American Society of Testing Materials (A.S.T.M.) index; for quantitative analysis the intensities of a few lines are best measured using scalers for the actual lines and chart recorders for the background. When carrying out an analysis using film mothods, an internal standard is necessary to furnish comparison lines; but this is unnecessary with a spectrometer, where the absorption of the sample can be measured readily. One of the features of the spectrometer, however, is that it is blind, and lines not suspected of being present can easily be missed. When examining completely unknown samples, a preliminary photograph is essential.

Dr. G. D. Rieck described the new 'Norelco' spectrometer in which the detecting Geiger counter is traversed in a vertical circle over a range of $38-165^{\circ}$, an angular accuracy of better than $0.01^{\circ}$ being achieved. The $\mathrm{X}$-ray optical system gives a very high resolution coupled with a low background. The $X$-ray tube is mounted vertically, and its output is well stabilized; both scaling and chart-recording facilities are offered, and a special instrument allows the intensities to be recorded automatically at chosen small intervals of the angular range with a constant statistical error, a valuable feature in the study of line profiles and for the detection of weak lines.

In a paper on recent developments in X-ray counters, Dr. U. W. Arndt said that, while Geiger counters are now readily available having adequate sensitivity and stability, they cannot be used for accurate work at intensities exceeding 1,000 counts/ sec. This rate can be increased about a hundred-fold with either proportional or scintillation counters. Moreover, with the former, though not with the latter, it is possible to make use of their energydiscriminating properties which allow an adequate degree of monochromatization to be obtained by the use of suitable pulse-selecting circuits. Dr. Arndt described a simply constructed argon-flow proportional counter, the long-term stability of which is superior to that of most Geiger counters. He also described some of the recent scaling and voltagestabilizing circuits which would considerably reduce the cost of equipment for counting; examples of these were shown in the exhibition.

Dr. G. E. Bacon reviewed the techniques used in neutron diffraction work on both single-crystal and powder samples. Crystal-reflected neutron beams of a wave-length of about 1 A. are employed, for which the most useful detector is a boron-10 trifluoride proportional counter. The main differences between neutron and X-ray spectrometers are due to the much larger size of the detector and the very feeble neutron intensity available, so that large samples 
must be used. Even so, counting-rates of 100 counts/ min. are rarely exceeded. Absolute intensity measurements are easier than with X-rays, since the scattering factor is independent of the scattering angle. In single-crystal work zero layer lines only are measured; in this type of work extinction corrections are generally considerable owing to the large crystals employed. Dr. W. Cochran briefly considered the use of counter methods for single-crystal X-ray measurements. Here overlapping spectra are not troublesome; lower resolution and lower accuracy of angle measurement are required than in powder work, and smaller spectrometer radii can thus be employed. Integrated reflexions can be measured conveniently by using a stationary crystal close to a very broad and uniform X-ray focus. Drs. Hirsch and Rieck pointed out the difficulty of obtaining the necessary uniformity of the focal spot.

The consideration of methods of identification by $\mathrm{X}$-ray crystallographic techniques was opened by Mr. H. S. Peiser, who pointed out how each problen requires different methods of approach. The enormous scope of X-ray identification methods justifies their use in nearly every manufacturing industry, for not only the constituents of a sample, but also their grain-size and nature, can often be identified by crystallographic methods.

Dr. D. M. C. McEwan described the special diffi. culties arising in the identification of clay minerals which may exist in the form of intimate mixtures on a molecular scale. Such structures can often be described only by their radial distributions. Frequently, the labile nature of clays causes line shifts to occur even with the simplest of preparatory treatments, and these shifts and the broadness of the bands render the standard card index inadequate. The most important information is often afforded by examining long spacings.

Special techniques are required in the identification of toxic and radioactive materials in order to safeguard the health of the operators and to prevent the fogging of the film. Some of these methods were described by Dr. J. Thewlis, who pointed out that in these cases minute quantities have to be used, the sample weighing less than a milligram. Photographic methods are employed, and the specimen is sealed in a capillary tube. With suitable precautions, samples having activities of several hundred microcuries can be photographed.

The most useful guide in the identification of most inorganic and some organic crystalline substances is the A.S.T.M. card index of lattice spacings, which now contains some five thousand cards, and the history of this index and of its continual growth and revision were related by Dr. A. J. C. Wilson, who has been in charge of the British part of this venture since 1942. A new type of index card is being produced which contains much more information than the old pattern. A set of 'Keysort' cards is now available, as are alphabetical and grouped indexes in book form. New and more accurate data are being substituted for the older information, spectrometers being used instead of powder cameras.

The need for supplementing information obtained from lattice spacings by other data was urged by Mr. R. Brooks. A consideration of the absorption of the sample, and of the type of fluorescent radiation it produces, often gives important clues, as do line broadening and the presence of preferred orientation. When single crystals are available axial lengths can be measured. Colour, refractive index, morphology and infra-red spectra may be of importance in addition to X-ray data.

Dr. K. W. Andrews said that he has found it more convenient at times to use one's own collection of known patterns than to employ the A.S.T.M. index for identification. In Mr. G. H. Cockett's experience, the use of lithium fluoride-reflected radiation is very valuable in identification problems, and he also makes frequent use of X-ray fluorescent analysis. Prof. $J$. D. Bernal suggested that the problems of analysis could be simplified by breaking up the complete A.S.T.M. index into groups of related substances; the weakest lines, the longest spacings, or 'Ionely lines' of a pattern are often more characteristic of a substance than the strongest line. Prof. E. G. Cox announced that he is investigating the best method of indexing single-crystal data for identification purposes.

A valuable feature of the conference was the unusually extensive exhibition. An informative and stimulating evening discourse was delivered by Dr. R. W. G. Wyckoff, whose subject was "A Survey of X-ray Diffraction in the United States". The next conference of the Group will be held in Cambridge during April 1-2, 1954, and will be devoted to modern structure analysis.

U. W. ARndt

\section{ANTIBIOTICS IN PIG FOOD}

TNTIL recently the use of antibiotics in animal feeds was prohibited in Great Britain; but the Penicillin Act, 1947, has now been amended by the Therapeutic Substances (Prevention of Misuse) Act, 1953 , and the Statutory Instrument 1953 No. 1174 Therapeutic Substances : The Therapeutic Substances (Supply of Antibiotics for Agricultural Purposes) Regulations, 1953-allows the use of aureomycin and penicillin in pig and poultry foods. The purpose of these additions is the improvement of growth of pigs and poultry under practical conditions, first observed in the United States ${ }^{1}$, where antibiotics have been used on a large scale as growth promoters for several years now. The Ministry of Agriculture has now issued Advisory Leaflet No. 418, "Antibiotics in Livestock Feeding" (H.M.S.O. 2d.).

In October 1951 the Agricultural Research Council called a meeting to decide whether the available findings justified the use of antibiotics in Britain. By that time, many American publications had confirmed the value of antibiotics; but British experimental evidence was still very limited ${ }^{2}$, and in view of the marked difference in British and American methods of pig feeding and management, further work here was thought necessary before a decision was taken. The Agricultural Research Council therefore organized further trials, which took place in 1952 , and $a$ summary of their results was published in July $1953^{3}$.

The trials, designed and analysed with the guidance of statisticians, were of two kinds. One was to determine the effect of antibiotic supplements on the growth-rate, efficiency of food conversion and carcass quality of fattening pigs, the other to measure their volue for suckling pigs.

Six research centres were asked to work with fattening pigs. They were: the Agricultural Research Council Field Station at Compton, the British Oil and Cake Mills, Ltd., Experimental Station at Stoke Mandeville, the Harper Adams Agricultural College, the National Agricultural Advisory Service Experi- 